\title{
Acolhimento em um serviço de emergência: percepção dos usuários
}

\author{
Embracement in an emergency service: users' perception \\ Acogimiento en un servicio de emergencia: percepciones de los usuarios
}

\section{Maria Vilani Cavalcante Guedes', Ana Ciléia Pinto Teixeira Henriques", Morgama Mara Nogueira Lima"l}

' Universidade Estadual do Ceará, Curso de Graduação em Enfermagem, Curso de Mestrado Acadêmico Cuidados Clínicos em Saúde. Fortaleza-CE, Brasil.

"Faculdade Metropolitana da Grande Fortaleza, Curso de Graduação em Enfermagem. Fortaleza-CE, Brasil.

\section{Submissão: 15-08-2011 Aprovação: 04-03-2013}

\section{RESUMO}

Objetivou-se compreender como os usuários de um serviço de emergência percebiam a atuação da enfermagem na realização do acolhimento. Foram realizadas entrevistas com 382 usuários do serviço, de outubro de 2008 a março de 2009 . Abordaramse, de maneira aleatória, sujeitos que haviam sido avaliados no acolhimento e encaminhados ao setor de emergência. Verificouse que a maioria dos usuários (34,5\%) referiu ter sido atendida pela equipe de enfermagem, porém menos da metade $(49,2 \%)$ disse ter sido orientada por algum profissional da equipe no acolhimento. As maiores dificuldades sentidas pelos usuários referiram-se à carência de informações e de respeito no atendimento. Ressalta-se a importância de maior atuação da enfermagem no acolhimento, entendido como uma atividade não restrita à porta de entrada do serviço.

Descritores: Acolhimento; Serviço Hospitalar de Emergência; Cuidados de Enfermagem; Enfermagem.

\section{ABSTRACT}

The objective was to understand how users of an emergency service see the nursing action in the user embracement. Interviews were carried out with 382 service users from October 2008 to March 2009. We randomly approached subjects that were assessed in the embracement and they directed to the emergency ward. It was verified that even though most users (34.5\%) reported having being assisted by the nursing team, few reported having been guided by some professional of the embracement team $(49.2 \%)$. The major difficulties experienced by users referred to the lack of information on care and respect in the assistance. We emphasize the importance of more nursing activities in user embracement, understanding it as an activity not restricted to a service entrance.

Key words: User Embracement; Emergency Service Hospital; Nursing Care; Nursing.

\section{RESUMEN}

El objetivo fue comprender como los usuarios de un servicio de emergencia percibían las actividades en el acogimiento. Se Ilevaron a cabo entrevistas con 382 usuarios del servicio, de octubre de 2008 a marzo de 2009. Se acercó a los sujetos de azar que fueron evaluados en la recepción y se habían dirigido al departamento de emergencia. Se encontró que la mayoría de los usuarios (34,5\%) declarou que había sido recibido por el equipo en el acogimiento, algunos dijeron haber sido guiados por un profesional del equipo en el acogimiento $(49,2 \%)$. Las principales dificultades que experimentan los usuarios fueron la falta de información y respeto en la atención. Se destaca la importancia de mayor actuación de la enfermería en el acogimiento, para comprenderse que esta actividad no se limita a la puerta de enlace del servicio.

Palabras clave: Acogimiento; Servicio de Urgencia en Hospital; Atención de Enfermería; Enfermería. 


\section{INTRODUÇÃO}

Mudanças na lógica de atendimento dos serviços para meIhorar a qualidade da assistência prestada são cada vez mais necessárias, especialmente nos serviços de urgência e emergência, cujas especificidades induzem os trabalhadores a se posicionar de maneira impessoal, com dificuldade de atuação de forma humanizada, em virtude de deficiências estruturais do sistema de saúde como um todo, alta demanda de atendimento, fragmentação do cuidado e ausência de filosofia de trabalho voltada para a humanização da assistência à saúde. Essa é uma demanda atual e crescente no contexto brasileiro que emerge da realidade em que usuários dos serviços de saúde queixam-se da qualidade do atendimento e, inclusive, de maus tratos ${ }^{(1)}$.

Para reorganizar os processos de trabalho em saúde e promover maior resolutividade das ações de saúde é necessário que as pessoas que buscam atendimento sejam acolhidas. Acolher ultrapassa a perspectiva de acesso ao serviço de saúde; é mais que uma ação de porta de entrada e recepção do usuário. É um processo contínuo que envolve sensibilidade e conhecimento técnico-científico dos profissionais para identificar necessidades de saúde derivadas de processos sociais, físico-biológicos, mentais e ambientais. É a responsabilização interessada e ativa pela condição de saúde do usuário que resulta em adequação do serviço de saúde ao perfil da população atendida.

O acolhimento é um modo de operar os processos de trabalho em saúde de forma a atender a todos que procuram pelos serviços de saúde, ouvir os pedidos dos pacientes e assumir uma postura capaz de escutar e identificar necessidades e pactuar respostas mais adequadas aos usuários. É uma tecnologia a ser estabelecida nos serviços de saúde em todos os níveis de complexidade. Implica prestar um atendimento com resolutividade e responsabilização, orientando, quando necessário, o paciente e sua família em relação a outros serviços de saúde para a continuidade da assistência, estabelecendo articulações para garantir a eficácia desses encaminhamentos ${ }^{(2)}$.

O acolhimento é uma ação tecno-assistencial que pressupõe a mudança da relação profissional/usuário e sua rede social, pautada em parâmetros técnicos, éticos, humanitários e de solidariedade, reconhecendo o usuário como sujeito e participante ativo no processo de produção da saúde ${ }^{(2)}$. Além do empoderamento do usuário pela produção de sua saúde, envolve a responsabilização do profissional pelo estado de saúde do usuário, um dos elementos essenciais para o efetivo acolhimento. Além disso, desperta no usuário um sentimento de confiança em relação ao profissional que presta assistência ${ }^{(3)}$.

O acolhimento significa a humanização do atendimento e pressupõe a garantia de acesso a todas as pessoas. Diz respeito ainda à escuta qualificada de problemas de saúde do usuário, visando fornecer sempre uma resposta positiva e responsabilizando-se pela resolução de seu problema. Em consequência, deve garantir a resolubilidade, objetivo final do trabalho em saúde, e solucionar efetivamente o problema do usuário(4) É mais que uma triagem qualificada ou uma escuta interessada. Pressupõe um conjunto formado por atividades de escuta, identificação de problemas e intervenções resolutivas para seu enfrentamento, ampliando a capacidade de a equipe de saúde responder às demandas dos usuários, reduzindo a centralidade das consultas médicas e utilizando o potencial dos demais profissionais ${ }^{(4)}$.

Percebe-se que o acolhimento colabora para a garantia de um atendimento de qualidade e humanizado, facilita a promoção da assistência integral, de forma que cada profissional possua uma visão holística do ser humano a ser atendido. Contudo, essa colaboração somente poderá existir se o acolhimento for entendido como um processo de corresponsabilidade de todos os profissionais pela saúde dos usuários, por meio do trabalho em equipe multiprofissional, qualificada e capacitada para tal, da postura acolhedora de todos e da liberdade para que se estabeleça o vínculo dos clientes com o serviço ${ }^{(5)}$.

Porém, o que se visualiza é que, tradicionalmente, a noção de acolhimento no campo da saúde tem sido identificada ora com uma dimensão espacial, que se traduz em recepção ágil e ambiente confortável, ora em uma ação administrativa de triagem e repasse de encaminhamentos para serviços especializados que, na maior parte das vezes, revela-se uma prática de exclusão social, na medida em que "escolhe" quem deve ser atendido(2).

Essa lógica tem produzido desestímulo dos profissionais, menor qualidade da capacitação técnica, pela falta inserção do conjunto de profissionais ligados à assistência, e a exclusão dos saberes que os usuários têm sobre sua saúde, seu corpo e seu grau de sofrimento, contradizendo todos os princípios estabelecidos para a realização efetiva do acolhimento ${ }^{(2)}$.

Serviços de urgência e emergência são permeados por condições complexas inerentes ao próprio ambiente e aos seres humanos que cuidam e são cuidados, que experienciam e vivenciam relações humanas também complexas no processo de cuidar/cuidado em um sistema organizacional hospitalar ${ }^{(6,7)}$. Assim, maior enfoque deve ser dado ao desenvolvimento das ações no acolhimento, compreendendo em que situações estão sendo desenvolvidos e de que forma os usuários visualizam a atuação dos profissionais de saúde nessa atividade, especialmente a equipe de enfermagem.

Esta, por seu papel atuante nas ações do acolhimento, deve se responsabilizar ativamente pela escuta ao usuário, compreendendo como deve posicionar suas ações nessa atividade para garantir a integralidade e universalidade, princípios defendidos pelo Sistema Único de Saúde.

Considera-se que o cuidado de enfermagem deve ir além da visão biológica e biomédica, de modo a integrar as diversas unidades e a multiplicidades dos seres. As ações do profissional de enfermagem em uma unidade de emergência precisam ser eficientes e eficazes; contudo, também precisam valorizar a subjetividade do ser humano ${ }^{(6)}$.

Protocolos têm sido utilizados para realização da classificação de risco, visando otimizar a decisão dos profissionais de saúde na priorização de atendimento dos usuários nos serviços de urgência e emergência, sendo um dos mais amplamente utilizados o Sistema de Triagem de Manchester (STM) que estabelece uma classificação de risco em cinco categorias. A partir da identificação da queixa principal do usuário pelo enfermeiro, um fluxograma orientado por discriminadores classifica o risco do paciente utilizando um sistema de cores: emergente (vermelho), muito urgente (laranja), urgente 
(amarelo), pouco urgente (verde) e não urgente (azul)(8-9).

Estudos apontam a importância da utilização de protocolos como este para mudanças no trabalho da atenção e produção da saúde, porém é importante enfatizar que outros aspectos devem ser considerados na realização do acolhimento nesses serviços, entre eles a percepção dos usuários quanto a essa ação tecnoassistencial(7-8,10).

Tendo em vista a complexidade envolvida nos processos de trabalho da equipe de enfermagem na realização do acolhimento nos serviços de emergência, este estudo buscou compreender como os usuários de um serviço de emergência percebem a atuação da enfermagem na realização do acolhimento.

\section{MÉTODO}

Realizou-se estudo descritivo, do tipo transversal, que envolve a coleta de dados em um ponto do tempo ${ }^{(11)}$, em unidade da emergência de um hospital de atenção secundária localizado em Fortaleza-CE.

Na ocasião, a capital do Ceará era dividida em seis secretarias regionais de saúde e a instituição cenário da pesquisa localizava-se na área administrativa da Secretaria Executiva Regional IV, que abrangia 19 bairros, com uma população estimada em 280 mil habitantes. Situada no bairro mais populoso da Regional IV, com aproximadamente 30 mil habitantes, era a segunda maior emergência do Estado do Ceará, com serviços de clínica médica, traumatologia e cirurgia, além de realizar exames laboratoriais complementares, eletrocardiograma (ECG) e raios $X^{(12)}$.

A pesquisa recebeu parecer favorável do Comitê de Ética em Pesquisa da Universidade Estadual do Ceará, sob protocolo $\mathrm{n}^{\circ}$ 07381232-0 e também foi autorizada pela direção da unidade de saúde. Foi integrada por 382 usuários do serviço que aceitaram participar voluntariamente do estudo após a explanação de todos os aspectos a serem abordados. Foram assegurados os aspectos de confidencialidade, autonomia e não maleficência, garantindo aos usuários que o atendimento não seria prejudicado em nenhum momento. Os que concordaram em participar do estudo assinaram um Termo de Consentimento Livre e Esclarecido que abordava todos esses aspectos para fins de registro legal, tanto para o usuário como para os pesquisadores.

A escolha dos sujeitos ocorreu de maneira aleatória, abordando aqueles que já haviam sido avaliados no acolhimento e encaminhados ao setor de emergência. Pacientes muito graves, que apresentavam perda do nível de consciência, dor de moderada a grave e distúrbios neurológicos em geral não foram convidados a participar a pesquisa pela impossibilidade de responder à entrevista.

A coleta de dados ocorreu por meio de entrevistas realizadas de outubro de 2008 a março de 2009, por pesquisadores capacitados. Foram guiadas por formulário com questões objetivas e subjetivas que compuseram 13 itens, sendo seis relacionados à caracterização sociodemográfica da amostra e outras sete a respeito do atendimento, com os seguintes questionamentos: tempo para realização do acolhimento; orientação recebida na chegada ao serviço; quem realizou essa orientação, se houve atendimento pela enfermagem e resolução dos problemas que o levaram ao serviço. Solicitou-se ainda ao usuário a atribuição de um conceito ao atendimento de enfermagem que lhe foi prestado e que informasse como gostaria de ser atendido na próxima vez que procurasse pelo serviço.

O tempo médio para aplicação dos instrumentos foi de 10 a 15 minutos, dependendo da possibilidade e da disponibilidade do usuário. Em nenhum momento o atendimento foi interrompido em decorrência da realização da entrevista.

$\mathrm{Na}$ análise dos dados, foi utilizada a estatística descritiva para as perguntas objetivas. Para as perguntas abertas, após leitura exaustiva das respostas, utilizou-se a técnica da análise temática, que consiste em identificar os núcleos de sentido que compõem a comunicação, procurando identificar seu significado para o estudo $^{(13)}$. Foram identificadas as seguintes temáticas: agilidade e critérios de atendimento; profissionais mais preocupados com a condição da pessoa; informações da Enfermagem sobre o fluxo de atendimento; ampliação da equipe para reduzir tempo de espera e respeito pelas pessoas, independentemente da condição social. As temáticas foram enriquecidas com os recortes mais marcantes dos discursos, para a compreensão do processo.

\section{RESULTADOS}

A caracterização sociodemográfica da amostra pesquisada foi reunida na tabela 1 , com as respectivas frequências e percentuais.

Tabela 1 - Caracterização sociodemográfica de usuários do serviço de emergência em um hospital de atenção secundária. Fortaleza-CE, 2009. $(\mathrm{N}=382)$

\begin{tabular}{|c|c|c|}
\hline $\begin{array}{c}\text { Características } \\
\text { sociodemográficas }\end{array}$ & $\mathbf{N}$ & $\%$ \\
\hline \multicolumn{3}{|l|}{ Sexo } \\
\hline Feminino & 213 & 55,8 \\
\hline Masculino & 169 & 44,2 \\
\hline \multicolumn{3}{|l|}{ Idade (anos) } \\
\hline$<20$ & 46 & 12 \\
\hline $21-40$ & 174 & 45,5 \\
\hline $41-60$ & 96 & 25,2 \\
\hline$>60$ & 66 & 17,3 \\
\hline \multicolumn{3}{|l|}{ Estado civil: } \\
\hline Casado & 188 & 49,2 \\
\hline Não casado* & 194 & 50,8 \\
\hline \multicolumn{3}{|l|}{ Escolaridade (anos) } \\
\hline Não estudou & 43 & 11,2 \\
\hline$\leq 10$ & 176 & 46,1 \\
\hline$>10$ & 163 & 42,7 \\
\hline \multicolumn{3}{|l|}{ Ocupação } \\
\hline Aposentado & 34 & 8,9 \\
\hline Empregado & 155 & 40,6 \\
\hline Estudante & 34 & 8,9 \\
\hline Autônomo & 90 & 23,6 \\
\hline Desempregado & 69 & 18 \\
\hline \multicolumn{3}{|c|}{ Renda familiar (em s.m. ${ }^{* *}$ ) } \\
\hline$\leq 1$ & 191 & 50,0 \\
\hline 1.1 a 3 & 157 & 41,1 \\
\hline 3.1 a 5 & 34 & 8,9 \\
\hline
\end{tabular}

* Nesta categoria incluem-se divorciados, viúvos e solteiros.

**S.M. = Salário mínimo, no período correspondente a R\$510,00. 
Na tabela 2 apresentam-se as características do atendimento e do acolhimento realizado na emergência do hospital, considerando aspectos da atuação da enfermagem.

Tabela 2 - Características do atendimento realizado no serviço de emergência de um hospital secundário. Fortaleza-

$$
\mathrm{CE}, 2009(\mathrm{~N}=382)^{*}
$$

\begin{tabular}{|c|c|c|}
\hline Características do atendimento & $\mathbf{N}$ & $\%$ \\
\hline \multicolumn{3}{|l|}{ Tempo de espera para atendimento } \\
\hline Imediatamente & 35 & 9,2 \\
\hline Até 1 hora & 179 & 46,8 \\
\hline 1 a 3 horas & 95 & 24,9 \\
\hline 3 a 5 horas & 37 & 9,7 \\
\hline$>5$ horas & 36 & 9,4 \\
\hline \multicolumn{3}{|l|}{ Orientação na chegada ao serviço } \\
\hline \multicolumn{3}{|l|}{ Sim } \\
\hline \multirow[t]{2}{*}{ Não } & 188 & 49,2 \\
\hline & 194 & 50,8 \\
\hline \multicolumn{3}{|l|}{ Quem realizou a orientação $(n=188)$} \\
\hline \multicolumn{3}{|l|}{ Médico } \\
\hline Enfermeira & 51 & 27,1 \\
\hline Porteiro & 48 & 25,5 \\
\hline Auxiliar/técnica & 28 & 14,9 \\
\hline Recepcionista/atendente & 17 & 9,0 \\
\hline Assistente social & 9 & 4,8 \\
\hline Guarda/maqueiro & 2 & 1,1 \\
\hline \multirow[t]{2}{*}{ Não soube informar } & 2 & 1,1 \\
\hline & 31 & 16,5 \\
\hline \multicolumn{3}{|l|}{ Atendimento pela equipe de enfermagem } \\
\hline \multicolumn{3}{|l|}{ Sim } \\
\hline \multirow[t]{2}{*}{ Não } & 331 & 86,6 \\
\hline & 51 & 13,4 \\
\hline \multicolumn{3}{|l|}{ Qualidade do atendimento $(\mathrm{n}=331)$} \\
\hline \multicolumn{3}{|l|}{ Excelente } \\
\hline Bom & 106 & 32,1 \\
\hline Regular & 67 & 20,2 \\
\hline Ruim & 102 & 30,8 \\
\hline \multirow[t]{2}{*}{ Péssimo } & 20 & 6,0 \\
\hline & 36 & 10,9 \\
\hline \multicolumn{3}{|l|}{ Resolução do problema } \\
\hline Sim & 250 & 65,4 \\
\hline Não & 132 & 34,6 \\
\hline
\end{tabular}

* Na tabela são explicitados $n$ diferentes quando há variação no número de participantes da pesquisa que responderam à questão.

Em face das opiniões e percepções dos pesquisados quanto ao acolhimento e às ações de enfermagem na emergência, as seguintes temáticas puderam ser organizadas e apresentadas com recortes dos discursos dos participantes da pesquisa.

\section{A. Agilidade e critérios de atendimento}

Nesta temática, os discursos dos usuários, em sua maioria, demonstraram o acolhimento como ferramenta principal para agilizar o atendimento e realizar uma avaliação inicial do risco e da gravidade dos pacientes que recorrem ao serviço. $\mathrm{O}$ acolhimento não atingiu ao objetivo organização das prioridades de assistência, visto que a grande maioria das queixas dos usuários se referiu a esse aspecto:
Deveria ser logo atendido os casos de emergência, com um serviço de triagem eficiente.

Consulta mais detalhada, melhor organização na fila e mais agilidade nos serviços.

Sabe-se que quando acometidas por dor ou mal-estar súbito, as pessoas sentem-se fragilizadas e consideram-se as mais necessitadas de atenção, em desacordo com o que preconiza a avaliação por risco.

B. Profissionais mais preocupados com a condição da pessoa

Em se tratando de uma ferramenta de práticas relacionais entre usuários e profissionais de saúde, as falas dos usuários agrupadas nesta temática permitiram perceber que existiam barreiras a serem vencidas quanto à humanização no atendimento dos serviços de emergência.

A grande demanda e a necessidade de agilidade no atendimento não permitem que ocorra um atendimento diferenciado, voltado para a totalidade do indivíduo e que, além de identificar processos fisiopatológicos, busque acolher o usuário, identificando as necessidades implícitas. As queixas de maus tratos, desrespeito e falta de humanidade foram as mais frequentes na avaliação dos discursos:

Que tivesse profissionais que trabalhassem mais preocupados com a dor e o sofrimento do outro.

Qualidade e quantidade de médicos, pois eles estão altamente robotizados e mecanizados... não dão um boa tarde...

Que tivesse profissionais que trabalhassem não pelo dinheiro, mas por amor à profissão.

O pessoal daqui precisa ser mais educado, de 1 a 100, $70 \%$ são ignorantes.

\section{Informações da Enfermagem sobre o fluxo do atendimento}

Tendo em vista a responsabilização pela continuidade do cuidado a que se propõe, o acolhimento, característico, mas não exclusivo das portas de entrada dos serviços, é responsável por direcionar para setores especializados os usuários que chegam ao serviço, de acordo com suas necessidades de saúde Os usuários que foram acolhidos pela equipe de enfermagem enumeraram algumas ações que lhes agradaram e outras que deveriam ser melhoradas. Reconheceram a contribuição dos enfermeiros no acolhimento humanizado, porém as falas também fizeram referência a falhas na orientação do fluxo do atendimento, para melhorar sua qualidade:

Fui recebido pela enfermeira, ela tirou minha pressão disse que estava alta e me mandou para o consultório do médico.

Que sempre chegasse alguém para olhar, para não ficar esperando sem saber o que vão fazer e se vão resolver o problema, como a enfermagem fez com um senhor. 
Uma pessoa na portaria que informasse com certeza para onde a gente deveria ir.

Se sempre tivesse a enfermeira, nós não 'ficava' sem saber o que fazer.

\section{Ampliação da equipe para reduzir tempo de espera}

A grande demanda nos serviços de emergência, motivada pela busca de resolução imediata dos problemas de saúde, alguns dos quais poderiam ser resolvidos na rede de Atenção Básica, resulta em superlotação e filas intermináveis, o que compromete a qualidade do atendimento aos usuários, que atribuem o problema à carência de profissionais.

A Enfermagem foi mencionada em muitas falas como a principal responsável pela organização e agilidade do atendimento, porém a figura do médico prevaleceu como necessária para maior agilidade na prestação da assistência, como percebido nas falas:

Mais profissionais que auxiliem o médico na organização do atendimento.

Mais rápido, mais enfermeiros no primeiro atendimento, pois demorou muito.

Gostaria que tivesse mais médicos e ser atendido com mais atenção, pois sou um senhor idoso de 76 anos e tenho que enfrentar uma fila imensa, sem prioridade no atendimento.

\section{E. Respeito pelas pessoas, independentemente de sua con- dição social}

A qualidade do atendimento nos serviços públicos de saúde foi sempre considerada inferior a dos serviços particulares. Os pacientes argumentavam que, por sua condição financeira, tinham como possibilidade de atendimento apenas os hospitais públicos, onde eram submetidos a uma assistência desumana, carente de respeito e atenção pelos profissionais. Percebeu-se nas falas dos usuários um sentimento de autodepreciação por sua condição socioeconômica e uma justificativa ao descaso sofrido devido a essa condição:

Esse descaso é por que sou pobre, se eu fosse rica a coisa era diferente.

Ter mais atenção com o paciente, pois a gente parece bicho, nem olham...

Queria ser tratada como gente, pois pago meus impostos.

\section{DISCUSSÃO}

O conhecimento da clientela atendida em serviços de saúde, especialmente os de urgência e emergência, é fundamental para o estabelecimento de prioridades e para o planejamento de ação dos gestores ${ }^{(14)}$, o que torna fundamental conhecer e descrever o perfil de usuários atendidos nos diferentes serviços de saúde.
As características do grupo amostral mantiveram estreita relação com a de estudos realizados em serviços de emergência e urgência ${ }^{(14)}$.Verificou-se a predominância de pacientes do sexo feminino $(55,8 \%)$, solteiros $(44,5 \%)$, com idade entre 21 e 40 anos (45,5\%), com até 10 anos de estudo $(46,1 \%)$ e renda familiar abaixo de três salários mínimos $(85,9 \%)$. Trata-se de uma população que caracteristicamente tende a procurar mais os serviços de saúde e que se encontra na faixa etária produtiva, com baixa escolaridade e condição socioeconômica precária.

Quanto às características do atendimento, observou-se que o tempo de espera foi breve após a chegada ao hospital, visto que 214 (56\%) pessoas foram atendidas em até uma hora e, dessas, $35(16,4 \%)$ o foram imediatamente após a chegada ao serviço. Entretanto, chamou atenção o fato de que 36 usuários passaram mais de 5 horas até receber atendimento, evidenciando dificuldades no atendimento frente à grande demanda do serviço. Estudos comprovam que a questão do acesso funcional é uma das principais dificuldades citadas pelos usuários e que pode resultar em uma avaliação negativa da qualidade do acolhimento nos serviços ${ }^{(3)}$.

A maioria dos usuários $(50,8 \%)$ referiu não ter recebido nenhuma orientação na chegada à emergência e queixaram-se da qualidade do acolhimento. As falas ressaltaram o papel do enfermeiro como direcionador e orientador no serviço, contribuindo para maior agilidade nos atendimentos e diminuição das grandes filas de espera.

A orientação fornecida ao usuário envolve a realização da escuta interessada, na qual o acolhimento deixa de ser apenas uma triagem de casos por gravidade e passa a ser a tomada de postura ética, que não pressupõe hora ou profissional específico para fazê-lo e implica compartilhamento de saberes, necessidades, possibilidades, angústias e invenções ${ }^{(2)}$.

Apesar do número expressivo de usuários que foram orientados ao chegar ao serviço (188), percebeu-se ainda uma atuação deficitária da equipe de enfermagem nesta ação, demonstrada pelo pequeno número de pacientes (65) que referiu ter recebido orientações de um profissional da equipe de enfermagem.

Quando questionados sobre o atendimento prestado pela equipe de enfermagem, 331 usuários afirmaram ter recebido atendimento por algum profissional da equipe, tendo a maior parte $(32,1 \%)$ atribuído conceito excelente ao atendimento recebido.

Ao atuar em uma unidade crítica de saúde, o profissional de enfermagem deve demonstrar destreza, agilidade, habilidade, bem como capacidade para estabelecer prioridades e intervir de forma consciente e segura no atendimento ao ser humano, sem esquecer que, mesmo na condição de emergência, o cuidado é o elo de interação/integração/relação entre o profissional e o usuário ${ }^{(6)}$.

No contexto da clínica ampliada, considera-se que o mais indicado seria articular o melhor da clínica à captação subjetividade por meio da escuta do sujeito envolvido na relação terapêutica no contexto social, o que poderia modificar a percepção dos usuários quanto à qualidade do serviço prestado $^{(15)}$. 
A resolubilidade do atendimento prestado foi confirmada por $65,4 \%$ das pessoas, o que revela que grande parte deixou o serviço sem que sua queixa fosse atendida. $O$ fato contradiz um dos principais princípios do acolhimento, ou seja, a postura de escuta e o compromisso de fornecer respostas às necessidades de saúde trazidas pelo usuário ${ }^{(2)}$.

Os usuários mencionaram ainda dificuldades sentidas em inúmeros outros serviços de saúde, como demora ou até mesmo atendimento inadequado por parte dos profissionais de saúde, área física inadequada, falta de material descartável, equipamentos e recursos humanos, o que causa indignação e descontentamento aos usuários, como aos profissionais de saúde ${ }^{(1)}$.

Porém, o mais marcante nos relatos referiu-se à falta de humanização no atendimento prestado pelos profissionais de saúde, destacando o respeito pelos indivíduos a despeito da condição social e da necessidade de profissionais mais preocupados com a pessoa. Isto demonstrou um grande entrave na realização de um acolhimento efetivo, baseado principalmente no vínculo que deveria ser estabelecido entre profissional e usuário.

Mesmo com todos os benefícios e as facilidades aos quais a tecnologia se propõe, muitos estudos demonstram que em algumas instituições o acolhimento tornou-se apenas mais um entrave entre o usuário e o trabalhador de saúde, uma barreira a mais a ser vencida em busca do atendimento ${ }^{(16)}$. Salienta-se que essas dificuldades não são sentidas apenas pelos usuários, mas também por profissionais que realizam o acolhimento ${ }^{(17)}$.

Pesquisa realizada avaliando o acolhimento em serviço de atenção secundária em Fortaleza-CE, segundo a percepção de enfermeiras, encontrou que a alta demanda desorganiza a estrutura de funcionamento do serviço, dificultando a acolhida, o vínculo e a comunicação entre os níveis da atenção ${ }^{(17)}$.

A centralidade da figura do médico também foi marcante nas falas que fizeram referência à necessidade de maior número de profissionais para prestar um atendimento adequado, o que refletiu a falha de compreensão de que nenhum profissional da saúde tem papel central no acolhimento, pois todos devem se responsabilizar pelas necessidades do usuário, o que torna os profissionais mais aptos para identificar os caminhos necessários à resolução dos problemas ${ }^{(18)}$.

A percepção limitada de que o acolhimento constitui apenas o processo de triagem de casos, selecionando os mais graves para o atendimento emergencial, pode ser caracterizada como um dos principais entraves para a implantação desta tecnologia nos serviços de saúde, dificultando ainda mais sua aceitação pela população, o que justifica a realização de estudos que visem a analisar, na visão do usuário, como o acoIhimento está sendo desenvolvido em diferentes realidades, especialmente nos serviços de emergência.

Torna-se fundamental a compreensão, não apenas por parte dos profissionais de saúde, mas também da população assistida, que o acolhimento implica prestar atendimento com resolubilidade e responsabilização, envolvendo além da avaliação da prioridade clínica, aspectos humanísticos e organizacionais fundamentais para produção de saúde nesses contextos $^{(17)}$.
A escuta ao usuário é considerada a maneira mais fiel de se analisar um programa e as respectivas ações nos espaços de saúde, visto que a melhoria da qualidade dos serviços é o foco principal do desenvolvimento dessas ações. A avaliação dos usuários permite ouvir sua opinião sobre os serviços em função de suas necessidades e expectativas e é uma das atividades que pode assegurar a qualidade dos serviços de saúde(4).

Empoderar a Enfermagem, levando-a a refletir sobre seu papel crucial na realização do acolhimento efetivo dos usuários de um serviço é fundamental para a valorização da equipe, tanto pelos próprios profissionais, quanto pelos usuários dos serviços. As falas demonstraram o quão importante é a atuação da equipe de enfermagem na realização do acolhimento, apesar do pequeno número de profissionais que foram referidos participando de algumas atividades, como a orientação na chegada ao serviço.

$\mathrm{O}$ acolhimento produz profundas mudanças no processo de trabalho dos profissionais não médicos. Assim, a enfermagem tem importante contribuição nesse novo modo de atendimento à demanda espontânea, devendo fortalecer sua atuação na consolidação do acolhimento como prática de transformação do processo de trabalho em saúde ${ }^{(19)}$. Os profissionais de enfermagem, assim como toda a equipe de saúde, devem estar sensibilizados, amparados e assistidos, para que as ações de acolhimento estejam presentes em toda a atenção prestada, e não apenas em um local específico, como se pertencessem a uma área física do serviço ${ }^{(6)}$.

O acolhimento, compreendido como uma tecnologia leve fundamentada nas relações, contempla a existência de um objeto de trabalho dinâmico, em contínuo movimento, não mais estático, passivo ou reduzido a um corpo físico. Esse objeto exige dos profissionais da saúde, especialmente do enfermeiro, uma capacidade diferenciada no olhar a ele concedido, a fim de que percebam a dinamicidade e a pluralidade de ações que desafiam os sujeitos à criatividade, à escuta, à flexibilidade e ao sensível(20).

É fundamental a compreensão, tanto por profissionais como por usuários, que mais que a realização de procedimentos e técnicas, a qualidade do cuidado baseia-se nas relações que são desenvolvidas e fortalecidas entre profissionais e usuários, verdadeiros parceiros na busca pela promoção da saúde.

\section{CONSIDERAÇÕES FINAIS}

O estudo permitiu reconhecer as dificuldades sentidas pelos usuários no atendimento em serviços de emergência, já amplamente descritas na literatura, e que denunciaram as condições desumanas a que são submetidos os usuários do Sistema Único de Saúde.

A visão do acolhimento realizado no serviço apresentou-se deturpada por antigos conceitos, como os de triagem, seleção, organização de filas, algo que deve ser melhor trabalhado pelos profissionais que realizam tais ações ainda de maneira isolada, o que desconfigura a real função do acolhimento como nova forma de organização das demandas nos serviços de saúde. 
A centralidade da figura do médico, apesar de ainda marcante em algumas falas, perde força à medida que se estabelece o papel do enfermeiro como orientador e facilitador no desenvolvimento de atividades do acolhimento, o que pôde ser constatado em algumas opiniões dos usuários.

Apesar das inúmeras deficiências estruturais encontradas nas emergências do país, inclusive na que constituiu o cenário desta pesquisa. As dificuldades mais relatadas foram carência de: atenção, humanização, respeito ao paciente e, muitas vezes, educação dos profissionais, demonstrando que a mudança de postura profissional, por meio de um simples olhar, toque, palavra de apoio e de orientação, é muito mais importante do que o remédio e o exame.

Portanto, transpor-se para o lugar do outro pode ser a grande resposta para o problema enfrentado por milhares de usuários dos serviços de saúde. Para isto, não são necessários grandes recursos, apenas conscientização e responsabilização para com o ser humano.

\section{REFERÊNCIAS}

1. Andrade LM, Martins EC, Caetano JA, Soares E, Beserra EP. Atendimento humanizado nos serviços de emergência hospitalar na percepção do acompanhante. Rev Eletr Enf [periódico na internet] 2009 [acesso em 15 jul 2011];11(1):151-7. Disponível em: http://www.fen.ufg.br/ revista/v11/n1/v11n1a19.htm.

2. Ministério da Saúde. Secretaria Executiva. Núcleo Técnico da Política Nacional de Humanização. Humaniza SUS: acolhimento com avaliação e classificação de risco - um paradigma ético-estético no fazer em saúde. Brasília: Ministério da Saúde; 2004.

3. Lima MADS, Ramos DD, Rosa RB, Nauderer TM, Davis R. Acesso e acolhimento em unidades de saúde na visão dos usuários. Acta Paul Enferm 2007;20(1):12-7.

4. Solla, JJSP. Acolhimento no sistema municipal de saúde. Rev Bras Saúde Matern Infant 2005;5(4):493-503.

5. Silva LG, Alves, MS. O acolhimento como ferramenta de práticas inclusivas de saúde. Rev APS 2008; 11(1):74-84.

6. Baggio MA, Callegaro GD, Erdmann AL. Compreendendo as dimensões de cuidado em uma unidade de emergência hospitalar. Rev Bras Enferm 2008;61(5):552-7.

7. Belluci Júnior JA, Matsuda LM. Construção e validação de instrumento para avaliação do acolhimento com Classificação de Risco. Rev Bras Enferm 2012;65(5):751-7.

8. Pinto Júnior D, Salgado PO, Chianca TCM. Validade preditiva do Protocolo de Classificação de Risco de Manchester: avaliação da evolução dos pacientes admitidos em um pronto atendimento. Rev Latino-Am Enferm [periódico na internet] 2012 [acesso 26 fev 2013];20(6):[08 telas]. Diponível em: < http://www.scielo.br/pdf/rlae/v20n6/pt_06.pdf.

9. Freitas P. Triagem no serviço de urgência: Grupo de Triagem de Manchester. 2.ed. Portugal: BMJ Publishing Group; 2002.

10. Souza CC, Toledo AD, Tadeu LFR, Chianca TCM. Classificação de risco em pronto-socorro: concordância entre um protocolo institucional brasileiro e Manchester. Rev.
Latino-Am. Enfermagem. [periódico na Internet]. 2011 [acesso 7 nov 2011];19(1):[08 telas]. Disponível em: http://www.scielo.br/pdf/rlae/v19n1/pt_05.pdf.

11. Polit DF, Beck CT, Hungler BP. Fundamentos da pesquisa em enfermagem: métodos, avaliação e utilização. 5. ed. Porto Alegre: Artmed; 2004.

12. Rede hospitalar de Fortaleza. Hospital Frotinha de Parangaba. [acesso em 14 jul 2009]. Disponível em: http:// www.sms.fortaleza.ce.gov.br/sms_v2/redes_redeHospitalar_FrotinhaParangaba.asp.

13. Bardin L. Análise de conteúdo. Lisboa: Edições 70; 2009.

14. Furtado BMASM, Araújo Jr JLC, Cavalcanti P. O perfil da emergência do Hospital da Restauração: uma análise dos possíveis impactos após a municipalização dos serviços de saúde. Rev Bras Epidemiol 2004; 7(3): 279-89.

15. Ramos DD, Lima MADS. Acesso e acolhimento aos usuários em uma unidade de saúde de Porto Alegre, Rio Grande do Sul, Brasil. Cad Saúde Pública 2003;19(1):27-34.

16. Souza ECF, Vilar RLA, Rocha NSPD, Uchôa AC, Rocha PM. Primary health care access and receptivity to users: an analysis of perceptions by users and health professionals. Cad Saúde Pública Rio de Janeiro 2008;24 Sup 1:S100-S110.

17. Mendonça FAC, Sampaio LRL, Linard AG, Silva RM, Sampaio LL. Acolhimento e vínculo na consulta ginecológica: concepção de enfermeiras. Rev Rene 2011;12(1):57-64.

18. Zanelatto DM, Dal Pai D. Práticas de acolhimento no serviço de emergência: a perspectiva dos profissionais de enfermagem. Cienc Cuid Saude 2010;9(2):358-365.

19. Takemoto MLS, Silva EM. Receptiveness and changes in the nursing work process in healthcare units in Campinas, São Paulo, Brazil. Cad Saúde Pública Rio de Janeiro 2007;23(2):331-40.

20. Rossi FR, Lima MADS. Acolhimento: tecnologia leve nos processos gerenciais do enfermeiro. Rev Bras Enferm 2005;58(3): 305-10. 